\title{
Acoustic Pressure and Particle Velocity for Spatial Filtering of Bottom Arrivals
}

\author{
Paulo Felisberto ${ }^{(1)}$, Paulo Santos ${ }^{(1)}$, and Sérgio M. Jesus, Member, IEEE
}

\begin{abstract}
This paper discusses the advantages of using a combination of acoustic pressure and particle velocity motion for filtering bottom arrivals. A possible area of application is reflection seismology where, traditionally, the seismic image is extracted from the bottom-reflected broadband acoustic signals received on hydrophones. Since hydrophones are omnidirectional in nature, the received bottom returns are often contaminated by waterborne signals, sea surface reflections, and noise. A substantial part of the processing of the data is dedicated to filtering out these unwanted signals. Today, vector sensors allow us to measure both acoustic pressure and particle velocity motion in a single and compact sensor. The combination of pressure and particle velocity measured at a single location or particle velocity and particle velocity gradient at closely spaced locations allows for spatial beam steering to predetermined directions and filter out unwanted replicas from other directions. Moreover, this can be done at the sensor level, dramatically decreasing the offline processing. The spatial filtering capabilities of various pressure-pressure, particle velocity-particle velocity, and pressure-particle velocity combinations are analyzed in view of filtering the bottom arrivals. It is shown that the combination of pressure and vertical particle velocity and, particularly, the combination of vertical particle velocity and particle velocity gradient enhance bottom arrivals. Moreover, a simple steering procedure combining pressure and particle velocity components of a triaxial sensor allows us to determine the tridimensional structure of the acoustic field and the separation of the bottom reflections. The spatial selectivity of the various sensor combinations is shown with simulations and verified with experimental data acquired with $10 \mathrm{~cm}$ separated vector sensors in the 800-1250-Hz band, during the Makai 2005 sea trial, off Kauai Island, HI, USA.
\end{abstract}

Index Terms-Geophysical signal processing, seismic measurements, spatial filtering, underwater acoustic vector sensor (VS).

\section{INTRODUCTION}

$\mathbf{O}$ CEAN bottom exploration represents a technological challenge and an important economic and scientific goal. The estimation of geological and geophysical parameters is related to a vast and diverse number of application fields, such as oil and gas exploration, the study of sediments and the upper

Manuscript received March 14, 2017; revised September 19, 2017; accepted February 15, 2018. Date of publication March 30, 2018; date of current version January 11, 2019. This work was supported by the European Union H2020 Research Program under WiMUST Project (Contract 645141). This paper was presented in part at the 2016 MTS/IEEE/OES OCEANS Conference, Monterey, CA, USA, September 19-22, 2016. (Corresponding author: Paulo Felisberto.) Associate Editor: G. Potty

The authors are with the Laboratory of Robotics and Systems in Engineering and Science, University of Algarve, Faro 8005-139, Portugal (e-mail: pfelis @ualg.pt; pjsantos@ualg.pt; sjesus@ualg.pt).

Digital Object Identifier 10.1109/JOE.2018.2807898 oceanic crust geophysics, or even sonar imaging of archaeological remains. The objective of seismic processing is to manipulate the acquired data into a seismic image that can be used to infer the sub-bottom detailed structure (number of layers, layer thickness, and sound velocities). The seismic image is obtained from the bottom-reflected replicas of signals generated by highpower impulsive sound sources, such as airguns or sparkers. Traditionally, marine seismic surveying is performed by a specially equipped vessel towing cables of hydrophones (known as streamers) that could be up to $8 \mathrm{~km}$ long. The streamers and the sources are deployed under the ocean surface, in precise geometries to facilitate the processing of acquired data [2]. The operation at sea of conventional seismic systems is costly and complex due to their size, weight, and geometric constraints. For seismic purposes, the streamers are 20 wavelengths long or more to give a directional resolution orthogonal to the direction of the array of one or two degrees. These constraints impose severe operational limitations in coastal areas.

Developments in sensor technology have led to a new generation of sensors - the vector sensors (VSs) [3] — which measure the three particle velocity components and the acoustic pressure (when a hydrophone is collocated with the particle velocity sensor). Particle velocity components along each of the three axes can be determined either by pressure gradient, using two omnidirectional hydrophones (whose distance should be at least four times smaller than the minimum wavelength [4]), connected as a dipole, or by using accelerometers (presently the most common solution). The spatial filtering capabilities of VS have become a subject of investigation in the 1990s [5], [6]. Most of the research involving VS is related to their capabilities for direction-ofarrival (DOA) estimation, clearly outperforming acoustic pressure only (scalar) hydrophones [7]. A single VS is able to estimate both elevation and azimuth angles. An array of vector sensors (VSA) gives an increased directivity gain that is not possible to achieve with an array of the same number of (scalar) hydrophones [8], [9]. Moreover, the direction of an impinging narrowband acoustic wave is obtained directly from the particle velocity measurements with an improved performance [10].

The influence of noise in underwater VS (or VSA) processing has been discussed by several authors (see, e.g., [11], [12]). D'Spain et al. [12] showed that the major disadvantage of a VS is related to the nonacoustic self-noise (motion-induced noise from vibration, electronic noise, etc.) on the particle velocity channels. It was also confirmed that additive combination of sensors is optimal for the detection of weak signals in presence of noise. Therefore, the use of VSAs appeared in several signal 


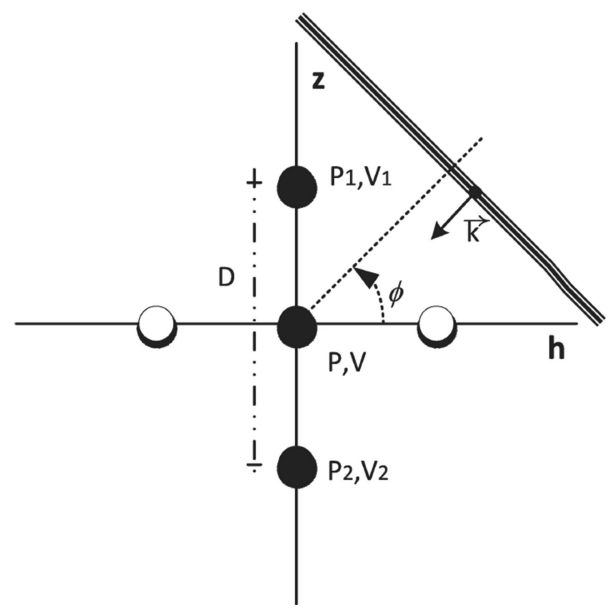

Fig. 1. Geometry definition: pressure sensors (to measure the pressure at each position, $P_{1}$ and $P_{2}$ ) and the (vertical) particle velocity sensors (to measure the particle velocity $V_{1}$ and $V_{2}$ ) are located along the vertical $(z)$ axis, separated by a distance $D . P$ and $V$ represent the pressure and the (vertical) particle velocity component at the origin. The horizontal $h$-plane is a combination of the $x$ - and $y$-axis in a 2-D scenario. The narrowband wavefront is characterized by the wave number vector $\vec{k}(k=|\vec{k}|=\omega / c, \omega=2 \pi f$ is the angular frequency; $c$ is the sound speed) and $\phi$ is the wavefront direction.

processing applications, using both simulated and experimental data, such as for source direction-finding and detection [13][15], source tracking [16], underwater communications [17], [18] and geoacoustic inversion [19], [20]. Using high-frequency signals in the $8-14-\mathrm{kHz}$ band, and a four element VSA, Santos et al. [19] demonstrated theoretically and verified with experimental data that a VSA provides higher estimation resolution of seabed parameters than a hydrophone array of same physical length. Experimental results also demonstrated that a spatially distributed array of gliders equipped with VS was able to track a source of opportunity such as a surface vessel, where surface noise could be efficiently rejected by using VS, outperforming current source localization methods [21].

The advantages of VS for seismic surveys were first showed by Lindwall [22]. In recent years, VS have also been used on towed streamers for the elimination of surface reflections (ghosts); however, details of these developments have limited publicity as they contain commercially sensitive information. It was also concluded that VS allowed us to operate surveys closer to the bottom, increasing the signal-to-noise ratio (SNR), hence enhancing the system seismic imaging performance [23], [24]. Recently, the usage of mobile platforms for seismic geophysical surveying, mainly those where the streamers are installed in AUVs, is subject of attention, in particular in the European project WiMUST [25]. The mobility of AUVs allows for the design of several receiving array configurations along the horizontal or the vertical axis, providing for such configurations to simultaneously cover shallow and deep water along the coast or in open ocean. To facilitate at sea operation, the VS can be embarked on an AUV, allowing for a significant reduction of energy and space requirements increasing autonomy and maneuverability.

This work follows a preliminary study for the development of a VS to be installed on AUVs for bottom imaging [1]. The advantages of a single VS in the suppression or separation of the direct and surface reflections and suppression of noise will be presented in the next sections. The spatial filtering capabilities of various combinations of VS channels, pressure, and particle velocity components are analyzed theoretically and with experimental data. Truly seismic data acquired by a VS were not available, thus the experimental data analysis is based on a subset of the Makai 2005 sea trial data, where signals in the band $0.5-1.5 \mathrm{kHz}$ were acquired by VSs at a short range $(<100 \mathrm{~m})$. Although the acquisition setup differs from that of a traditional seismic survey, the understanding gained could be extended to seismic systems design and signal processing.

This paper is organized as follows. Section II reviews the theory related to the combination of pressure and particle velocity given by pressure gradient and by particle velocity gradient. Section III is dedicated to the analysis of the influence of noise and SNR gain for the various VS combinations presented in Section II. Section IV shows through simulations the spatial selectivity of the various combinations of pressure and particle velocity for broadband signals. A real data example drawn from the Makai 2005 sea trial is given in Section V and, finally, Section VI draws the conclusions of this paper.

\section{Combinations of Pressure and Particle Velocity}

This section discusses the various additive combinations of pressure and/or particle velocity measurements to determine directional acoustic field components in view of seismic scenarios. The additive combination of pressure and particle velocity measurements is optimal to improve low SNR signals, which is the case of reflections from deep bottom layers in seismic applications. These combinations are particular cases of the wellknown super directive beamformers [12] and VS combinations discussed in [26].

\section{A. Particle Velocity Measurements}

From the linearized acoustic (Euler's) momentum equation, the acoustic pressure $p$ and particle velocity $\mathbf{v}$ are related by

$$
-\nabla p=\rho \frac{\partial \mathbf{v}}{\partial t}
$$

where $t$ represents the time variable and $\rho$ is the density.

Therefore, for sinusoidal motion at frequency $\omega$, one can write

$$
\left\{\begin{aligned}
V_{x}(\omega) & =-\frac{1}{j \rho \omega} \frac{\partial P(\omega)}{\partial x} \\
V_{y}(\omega) & =-\frac{1}{j \rho \omega} \frac{\partial P(\omega)}{\partial y} \\
V_{z}(\omega) & =-\frac{1}{j \rho \omega} \frac{\partial P(\omega)}{\partial z}
\end{aligned}\right.
$$

where $P(\omega), V_{x}(\omega), V_{y}(\omega)$, and $V_{z}(\omega)$ are the Fourier transform of pressure and of $x, y$, and $z$ particle velocity components, respectively. To combine (or compare) the pressure and particle velocity, both should be in the same units. According to the definition of acoustic impedance, the magnitudes of pressure and particle velocity are related by $p / v=\rho c$ under the plane wave approximation, where $c$ is the sound speed. Therefore, scaling the particle velocity by $\rho c$ allows us to define particle velocity in pressure units, the so-called pressure-equivalent 
particle velocity. In the rest of the paper, the term particle velocity implies pressure-equivalent particle velocity, unless otherwise stated.

The particle velocity component along a given axis can be estimated by the pressure gradient using two closely spaced hydrophones or by an accelerometer. When an accelerometer is used, the well-known relation between velocity $V$ and acceleration $A$ for a narrowband signal at frequency $\omega, A(\omega)=j \omega V(\omega)$, allows us to write the pressure-equivalent particle velocity as

$$
V(\omega)=\frac{\rho}{j k} A(\omega)
$$

To estimate the particle velocity from the pressure gradient, two pressure sensors aligned with the vertical axis at spacing $D$ are used, considering $P_{1}$ and $P_{2}$ the pressure signals in the frequency domain at each position (see Fig. 1). Without loss of generality, only the estimation of the vertical component $z$ of the particle velocity will be considered. The origin is in the middle point between the two pressure sensors and the angle $\phi$ of the incoming wave is defined relative to the normal of the sensors' axis. The estimated gradient by the first-order pressure difference is given by

$$
\frac{P_{1}(\omega)-P_{2}(\omega)}{D}=\frac{P(\omega) e^{j \omega \frac{D}{2 c} \sin (\phi)}-P(\omega) e^{-j \omega \frac{D}{2 c} \sin (\phi)}}{D} .
$$

When the spacing between pressure sensors $D$ is much smaller than the minimum wavelength $\lambda$, the pressure gradient (4) can be approximated by

$$
\frac{P_{1}(\omega)-P_{2}(\omega)}{D} \approx j k \sin (\phi) P(\omega)
$$

where $k=\omega / c$ is the amplitude of the wave number vector $\vec{k}$, therefore $k \sin (\phi)$ is the projection of the wave number vector onto the sensor's axis. As a rule of thumb, a separation $D$ smaller than one-fourth or less of the minimum wavelength should be considered. However, extremely short distances should be avoided because small differences in phase characteristics (offsets) in the individual sensors lead to large errors at low frequencies.

From (2), the particle velocity estimate along the $(z)$ axis in the frequency domain, $V_{z}(\omega)$, using the first-order differential approximation, is given by

$$
\begin{aligned}
V_{z}(\omega) & \approx-\frac{1}{j \rho \omega} \frac{P_{2}(\omega)-P_{1}(\omega)}{D} \rho c, \\
& \approx \frac{1}{j k} \frac{P_{1}(\omega)-P_{2}(\omega)}{D} .
\end{aligned}
$$

Then, combining (5) and (6) allows for obtaining the relation between pressure $P(\omega)$ and particle velocity $V_{z}(\omega)$ as

$$
V_{z}(\omega) \approx P(\omega) \sin (\phi)
$$

where it is assumed that the pressure and the particle velocity sensors are collocated.

It should be remarked that the particle velocity estimated from pressure measurements (6) or acceleration measurements (3) is frequency dependent, with a fall off rate of $6 \mathrm{~dB} /$ octave in both cases.

\section{B. Combining Pressure and Particle Velocity}

Pressure and particle velocity can be linearly combined using (7) to obtain $\tilde{P}(\omega)$ as

$$
\tilde{P}(\omega)=P(\omega)+V_{z}(\omega)=P(\omega)(1+\sin (\phi))
$$

where pressure and particle velocity have equal weight in the expression. In case the particle velocity is estimated by two closely located pressure sensors where $D \ll \lambda$, the pressure at the middle point between the sensors can be estimated by averaging as

$$
\frac{P_{1}(\omega)+P_{2}(\omega)}{2} \approx P(\omega)
$$

and combining (6) with (9) in (8), $\tilde{P}(\omega)$ can be written as

$$
\tilde{P}(\omega)=\frac{P_{1}(\omega)+P_{2}(\omega)}{2}+\frac{1}{j k} \frac{P_{1}(\omega)-P_{2}(\omega)}{D} .
$$

In case the particle velocity is measured by an accelerometer, acoustic pressure may be measured by a collocated hydrophone; in that case, the first term of the summation above is replaced by the hydrophone output $P(\omega)$ and the second term is replaced by (3).

\section{Particle Velocity Gradient}

When two particle velocity measurements, $V_{1}(\omega)$ and $V_{2}(\omega)$, are obtained at close locations (say with a separation $D$, see Fig. 1), one can estimate the (middle point) particle velocity gradient, following the same analysis as for the pressure gradient. Thus

$\frac{V_{1}(\omega)-V_{2}(\omega)}{D}=\frac{V_{z}(\omega) e^{j \omega \frac{D}{2 c} \sin (\phi)}-V_{z}(\omega) e^{-j \omega \frac{D}{2 c} \sin (\phi)}}{D}$

or for $D \ll \lambda$

$$
\frac{V_{1}(\omega)-V_{2}(\omega)}{D} \approx j k \sin (\phi) V_{z}(\omega) .
$$

Therefore, the relation between pressure and particle velocity gradient is given by

$\bar{V}_{z}(\omega) \doteq \frac{1}{j k} \frac{V_{1}(\omega)-V_{2}(\omega)}{D} \approx V_{z}(\omega) \sin (\phi)=P(\omega) \sin ^{2}(\phi)$.

For the particle velocity itself and for close-spaced sensors, one can write

$$
\frac{V_{1}(\omega)+V_{2}(\omega)}{2} \approx V_{z}(\omega) .
$$

The linear combination of particle velocity and particle velocity gradient is derived as

$$
\begin{aligned}
\tilde{V}_{z}(\omega) \doteq & V_{z}(\omega)+\bar{V}_{z}(\omega)=\frac{V_{1}(\omega)+V_{2}(\omega)}{2} \\
& +\frac{1}{j k} \frac{V_{1}(\omega)-V_{2}(\omega)}{D} \approx P(\omega)\left(\sin \phi+\sin ^{2}(\phi)\right) .
\end{aligned}
$$

So far, the dependence of various pressure and particle velocity combinations on the direction of the incident wave was 


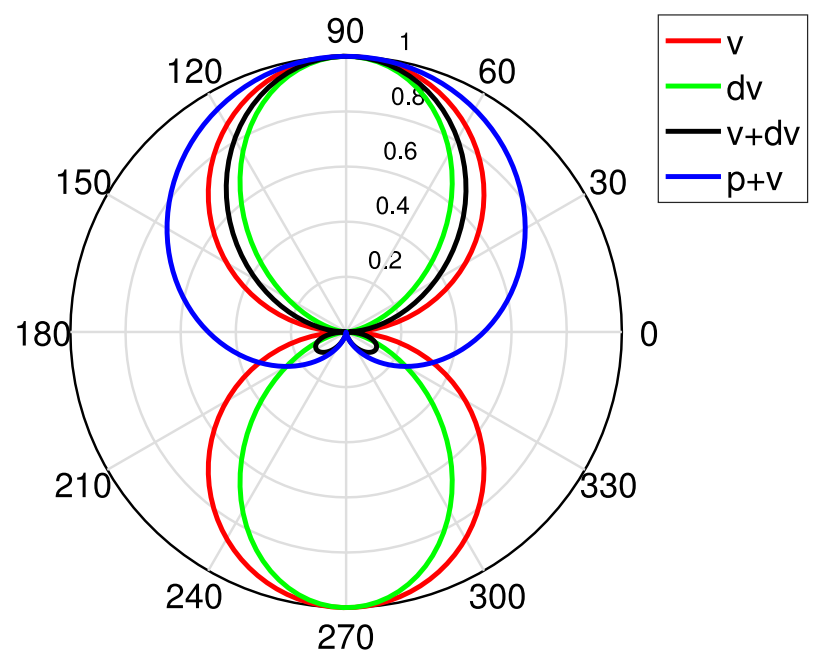

Fig. 2. Normalized amplitude directivity patterns for a pair of sensors placed along the vertical axis $\left(90^{\circ}\right)$ separated by $D=\lambda / 4$ : particle velocity [see (6), red], particle velocity gradient [see (13), green], combination of particle velocity and particle velocity gradient [see (15), black] and combination of pressure and particle velocity [see (8) and (10), blue].

discussed, which are often illustrated using directivity pattern curves.

\section{Directivity Pattern}

The spatial filtering characteristics of sensors and sensor arrangements are often described by normalized amplitude directivity patterns (or radiation diagrams) as $|B(\omega, \phi)| / \max (|B(\omega, \phi)|)$, where $B(\omega, \phi)$ is the beam pattern for each of the previous pressure and/or particle velocity combinations. Fig. 2 compares the normalized amplitude directivity patterns (unitary gain at maximum response) for particle velocity using (6) (red), particle velocity gradient only using (13) (green), combination of particle velocity and particle velocity gradient (15) (black), and a combination of pressure and particle velocity (8) [and (10)] (blue). In this example, the two sensors are aligned with the vertical axis $\left(90^{\circ}\right)$ and the angle $\phi$ is defined according with Fig. 1. The separation between sensors $D$ is assumed to be $\lambda / 10$. The derivative approximations by finite differences [see (6), (10), (13), and (15)] are frequency dependent and longer separations give rise to errors. It can be seen that particle velocity and particle velocity gradient describe a "dipole"-like directivity pattern, whereas the combination of particle velocity and particle velocity gradient or the combination of pressure and particle velocity show a "cardiod"-like directivity pattern. Considering a scenario of a geoacoustic survey, a combination of particle velocity sensors aligned with the vertical axis allows us to cancel (or significantly attenuate) the direct and surface-reflected paths, usually considered as a nuisance. Thus, both options are good candidates for spatial selectivity of incoming waves from a given direction.

The normalized amplitude directivity patterns presented in Fig. 2 consider only ideal sensors, where gain and phase of the sensors are equal. However, in real systems, due to a variety of reasons (sensors' sensitivity, electronics interface, sensors assembling or cabling), these parameters may vary from sensor to sensor. Particularly, in systems where accelerometers and

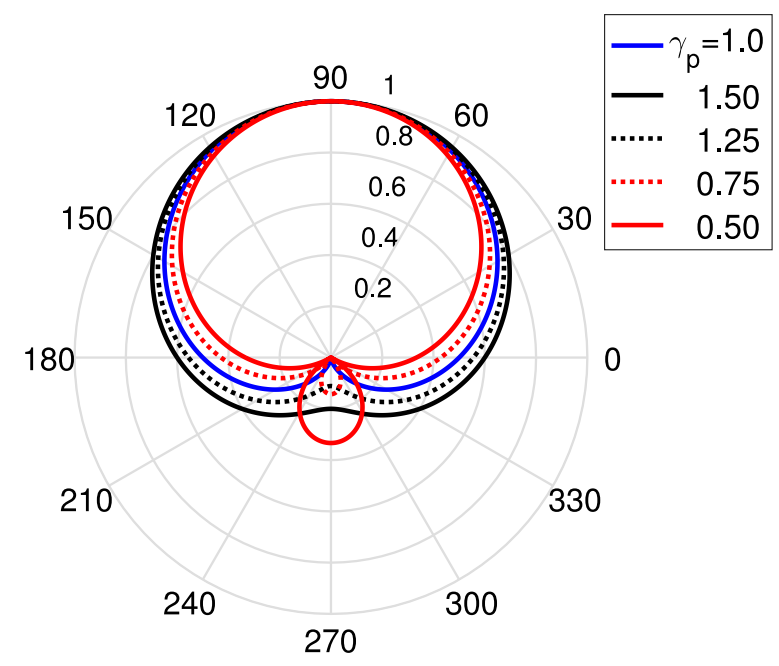

Fig. 3. Normalized amplitude directivity patterns of pressure and particle velocity combination for different pressure weights $\left(\gamma_{p}\right)$ [see (16)].

hydrophones are used, these issues introduce a mismatch, making practical calibration a challenge for the devices concerned. Equation (8) can be modified to accommodate these differences as follows:

$$
\tilde{P}(\omega)=\gamma_{p} P(\omega)+V_{z}(\omega)=P(\omega)\left(\gamma_{p}+\sin (\phi)\right)
$$

where $\gamma_{p}$ represents the weight of the pressure channel relative to the particle velocity channel. The dependence of the directivity pattern on $\gamma_{p}$ for the combination of pressure and particle velocity is shown in Fig. 3, where all responses were normalized by the maximum.

Fig. 3 shows that for $\gamma_{p}>1$, i.e., prevailing the pressure channel, the suppression capability of signals arriving from the opposite hemisphere is reduced (solid and dotted black lines). However, when the contribution of the pressure decreases $\gamma_{p}<1$, a secondary lobe appears in the opposite direction of the maximum response (solid and dotted red lines), but the main lobe is narrower and improves the suppression capabilities from intermediate directions in the hemisphere of the maximum response. These desirable characteristics are obtained at the expenses of a larger secondary lobe. Although $\gamma_{p}$ represents an "error correction" parameter, it can also be used as a tuning parameter for the data processing of a seismic source-receiver setup.

\section{E. Steering the Axis of Maximum Response}

The various combinations of pressure and vertical particle velocity measurements discussed above gave the maximum response at $90^{\circ}$ (or equivalently at $-90^{\circ}$ ). However, if particle velocity measurements are available simultaneously along various (noncollinear) axis, the axis of maximum response can be steered to a given direction. In the two-dimensional (2-D) case, this can be accomplished if the particle velocity measurements are available in both the vertical $(z)$ and the horizontal ( $x$ and $y$ ) planes, which is readily available in actual accelerometer-based VS. Then, the combination of pressure and particle velocity steered to direction $\phi_{0}$ is given by

$$
\tilde{P}\left(\omega, \phi_{0}\right)=P(\omega)+V_{h}(\omega) \cos \left(\phi_{0}\right)+V_{z}(\omega) \sin \left(\phi_{0}\right)
$$




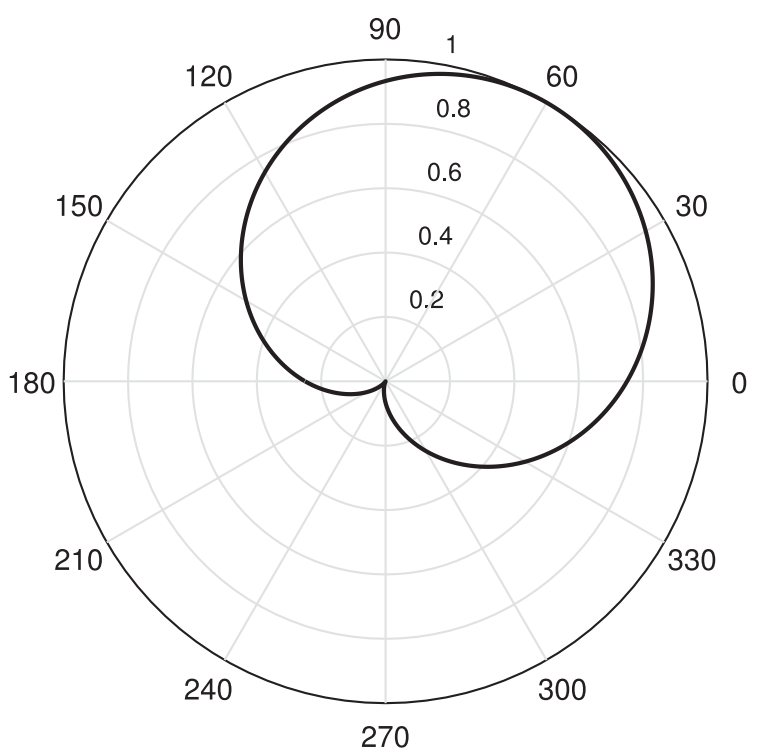

Fig. 4. Normalized amplitude directivity pattern of the combination of pressure, vertical and horizontal particle velocities steered to $60^{\circ}$ [see (17)].

where $V_{h}(\omega)$ and $V_{z}(\omega)$ are the horizontal and the vertical particle velocity measurements, respectively.

Fig. 4 shows the normalized amplitude directivity pattern combining pressure with vertical and horizontal particle velocity steered to $60^{\circ}$. Likewise, if particle velocity measurements in the three orthogonal axes $V_{x}(\omega), V_{y}(\omega)$, and $V_{z}(\omega)$ are available, then by extending (17), one can steer the axis of maximum response to any azimuth $\theta_{0}$ and elevation $\phi_{0}$ using

$$
\begin{aligned}
\tilde{P}\left(\omega, \theta_{0}, \phi_{0}\right)= & P(\omega)+V_{x}(\omega) \cos \left(\theta_{0}\right) \cos \left(\phi_{0}\right) \\
& +V_{y}(\omega) \sin \left(\theta_{0}\right) \cos \left(\phi_{0}\right)+V_{z}(\omega) \sin \left(\phi_{0}\right) .
\end{aligned}
$$

Steering the directivity patterns of a combination of pressure and particle velocity components is a simple and straightforward procedure that can also be used to compensate for sensor tilt due to platform motion or to focus on a particular DOA. Steering the directivity pattern of a combination of particle velocity and particle velocity gradient is also possible if the underlying measurements are available in two or three orthogonal axes. However, these are much more complex, and require two accelerometers along each axis. Moreover, the steering procedure involves nonlinear operations as division among components and frequencies. Such operations are very sensitive to noise and should be avoided in practice, thus steering the combination of particle velocity and particle velocity gradient will not be considered hereafter.

\section{Vector SENSOR Gain of the VARious Sensor COMBINATIONS}

When considering random signals received on closely spaced sensors, such as those used here for implementing VS, one has to consider the possibility of mutually correlated ambient noise in the acoustic pressure and particle velocity channels, as well as, generally uncorrelated sensor electronic noise. For the considered application of seismic processing, it is important to compare the VS SNR performance, with that of pressure only sensors (hydrophones), which are commonly used in seismic systems. To do so, the approach followed in this section is to compare the output $\mathrm{SNR}$ ( $\mathrm{SNR}_{\text {out }}$ ) of the various combinations of pressure and particle velocity discussed above to the pressure sensor only case $\left(\mathrm{SNR}_{\mathrm{ps}}\right)$. For simplicity, the analysis is restricted to the narrowband case at frequency $\omega$, the noise is assumed to be additive, zero mean, wide sense stationary, and uncorrelated with the signal in all channels of the VS output. Therefore, for a single VS, we analyzed a measure similar to array gain, herein defined as $\mathrm{SNR}_{\text {out }}=\mathrm{VSG} * \mathrm{SNR}_{\mathrm{ps}}$, where VSG is the so-called vector sensor gain.

The SNR of a (single) pressure sensor is given by $\sigma_{s}^{2} / \sigma_{n}^{2}$, where $\sigma_{s}^{2}$ is the signal power and $\sigma_{n}^{2}$ is the noise power (variance). Under these assumptions, the SNR can be approximated by $S_{s}(\omega) / S_{n}(\omega)$, where $S_{s}(\omega)$ is the power spectral density (PSD) of the signal and $S_{n}(\omega)$ is the PSD of the noise. The various combinations of sensors are linear; therefore, under the assumptions of uncorrelated signal with noise and zero mean noise, the power output can be computed independently for signal and noise, and the SNR at the output is given as the ratio of output signal power (variance) to output noise power (variance). For the noise analysis of the various combinations of pressure and particle velocity, it is important to consider the sensors' nonacoustic self-noise (thermal or electronic noise). The nonacoustic self-noise is, in general, modeled as white, both in time and space. The acoustic noise has, in general, a more complex spatio-temporal structure; therefore, for output noise power assessment, it is necessary to a priori know the intersensor and space-time cross correlation, respectively, cross-power density structure of the noise, which is dependent on the considered noise model. Several authors have studied the space-time structure of various underwater noise models not only for pressure [27]-[29], but also for pressure-particle velocity [11], [30], [31]. Table I summarizes the correlation functions for 3-D isotropic noise and for the narrowband directional noise model presented in [31], for the cases of collocated sensors and sensors lying in the vertical axis at a distance $D$. The 3-D isotropic noise is often used for ambient-noise modeling, while the narrowband directional noise can be used to model distant tonal propeller noise.

\section{A. Particle Velocity}

Using (6), the variance of the particle velocity obtained by pressure difference can be written as

$\mathrm{E}\left\{V_{z}(\omega) V_{z}^{*}(\omega)\right\}$

$=\frac{1}{(k D)^{2}} \mathrm{E}\left\{P_{1}(\omega) P_{1}^{*}(\omega)+P_{2}(\omega) P_{2}^{*}(\omega)-2 \operatorname{Re}\left\{P_{1}(\omega)\left(P_{2}^{*}(\omega)\right\}\right\}\right.$

where $\mathrm{E}\{\}$ is the mathematical expectation, $*$ represents the complex conjugation, and $P_{1}(\omega)$ and $P_{2}(\omega)$ are the pressure measurements (see Fig. 1). When the noise is uncorrelated be- 
TABLE I

SpATIAL NOISE CORRELATION Function FOR 3-D ISOTROPIC NOISE AND NARROWBAND DiRECTIONAL Noise From [31]

\begin{tabular}{|c|c|c|c|c|}
\hline \multirow[b]{2}{*}{ Noise model } & \multicolumn{3}{|c|}{$K_{x-y}\left(\mathbf{r}_{1}, \mathbf{r}_{2}\right)$} & \multirow[b]{2}{*}{$\mathrm{p}-\mathrm{v}_{z}$} \\
\hline & dist. & p-p & $\mathrm{v}_{z}-\mathrm{v}_{z}$ & \\
\hline $\begin{array}{l}\text { 3-D } \\
\text { isotropic }\end{array}$ & $\begin{array}{l}0 \\
\mathrm{D}\end{array}$ & $\begin{array}{c}1 \\
\operatorname{sinc}(k D)\end{array}$ & $\left(\frac{1}{k D} J_{1}(k D)^{\frac{1}{3}}-J_{2}(k D)\right)$ & $\begin{array}{c}0 \\
j J_{1}(k D)\end{array}$ \\
\hline $\begin{array}{l}\text { Narrowband } \\
\text { at elevation } \phi_{n}\end{array}$ & $\begin{array}{l}0 \\
D\end{array}$ & $\begin{array}{c}1 \\
e^{-j k D \sin \left(\phi_{n}\right)}\end{array}$ & $\begin{array}{c}\left(\sin \left(\phi_{n}\right)\right)^{2} \\
\left(\sin \left(\phi_{n}\right)\right)^{2} e^{-j k D \sin \left(\phi_{n}\right)}\end{array}$ & $\begin{array}{c}\sin \left(\phi_{n}\right) \\
\sin \left(\phi_{n}\right) e^{-j k D \sin \left(\phi_{n}\right)}\end{array}$ \\
\hline
\end{tabular}

$S_{n}(\omega)$ is the PSD of the noise pressure field. $K_{x-y}\left(\mathbf{r}_{1}, \mathbf{r}_{2}\right)$ gives the intersensor spatial structure of the noise between sensors of type $x$ and $y$, respectively, located at position $\mathbf{r}_{1}$ and $\mathbf{r}_{2}$. Particle velocity refers to pressureequivalent particle velocity. $\phi_{n}$ represents the elevation angle of the noise source. This table considers only collocated sensors (distance 0 ) and sensors separated by distance $D$ in the vertical axis; $J_{1}(\cdot), J_{2}(\cdot)$ represent spherical Bessel functions order 1 and 2, respectively.

tween sensors, the output noise power is

$$
S_{e, \text { out }}(\omega)=\frac{2 S_{e}(\omega)}{(k D)^{2}}
$$

where $S_{e}(\omega)$ is the PSD of the nonacoustic (electronic and thermal) self-noise of a single pressure sensor. It can be seen that the output noise power is frequency dependent $(k=\omega / c)$. If $S_{e}(\omega)$ is frequency independent (white noise), the output noise power increases $6 \mathrm{~dB} /$ octave as the frequency decreases. The noise power increase is also valid for accelerometer-based measurements of particle velocity, and can be a limitation, particularly for the detection of low-frequency signals [4], [12].

When the pressure is a plane wave of PSD $S(\omega)$ (where $\left.S(\omega)=\mathrm{E}\left\{P_{i}(\omega) P_{i}^{*}(\omega)\right\}, i=\{1,2\}\right)$, impinging in the sensor's axis at elevation angle $\phi$, using (19) and the pressureequivalent particle velocity (7), it is straightforward to obtain

$$
\mathrm{E}\left\{V_{z}(\omega) V_{z}^{*}(\omega)\right\}=\sin ^{2}(\phi) S(\omega)
$$

which applies for the signal and for the directional noise as well. Therefore, under these conditions, the SNR at the output is given by

$$
\mathrm{SNR}_{\mathrm{out}}=\frac{\sin ^{2}\left(\phi_{s}\right)}{\sin ^{2}\left(\phi_{n}\right)} \frac{S_{s}(\omega)}{S_{n}(\omega)}=\frac{\sin ^{2}\left(\phi_{s}\right)}{\sin ^{2}\left(\phi_{n}\right)} \mathrm{SNR}_{\mathrm{ps}}
$$

where $S_{s}(\omega), S_{n}(\omega), \phi_{s}$, and $\phi_{n}$ represent the PSD and the elevation angles of the signal and the noise, respectively, and $\mathrm{SNR}_{\mathrm{ps}}$ is the SNR of the single pressure sensor. One can conclude that in presence of distant directional noise, which generally reaches the receiver at low elevation angles, the SNR is improved, particularly for signals with steeper elevation angles. This applies when the power of the nonacoustic self-noise can be neglected, i.e., $S_{e}(\omega) \ll S_{n}(\omega)$. This assumption will be considered hereafter, unless stated otherwise.

Let us now consider the 3-D isotropic noise case. From (19) and the correlation functions in Table I, one can write

$$
\begin{aligned}
\mathrm{E} & \left\{V_{z, n}(\omega) V_{z, n}^{*}(\omega)\right\} \\
& =\frac{1}{(k D)^{2}}\left\{2 S_{n}(\omega)-\left[S_{n}(\omega)+S_{n}^{*}(\omega)\right] \operatorname{sinc}(k D)\right\} \\
& =\frac{2 S_{n}(\omega)}{(k D)^{2}}[1-\operatorname{sinc}(k D)]
\end{aligned}
$$

where $V_{z, n}(\omega)$ represents the Fourier transform of a sample of the vertical particle velocity noise component, since PSD is real $S_{n}^{*}(\omega)=S_{n}(\omega)$.

Using a Taylor expansion, it can be shown that for small $k D$, $(1-\operatorname{sinc}(k D)) /(k D)^{2} \approx 1 / 6$. Therefore, for the 3-D isotropic case, one can write

$$
\mathrm{SNR}_{\mathrm{out}} \approx \frac{\sin ^{2}\left(\phi_{s}\right) S_{s}(\omega)}{3 S_{n}(\omega)}=\frac{\sin ^{2}\left(\phi_{s}\right)}{3} \mathrm{SNR}_{\mathrm{ps}} .
$$

One can conclude that signals arriving from steeper angles show an improved SNR compared to those arriving from directions normal to the axis of the pressure sensors.

So far particle velocity measurements from pressure difference were considered, but when the sensor measures the particle velocity directly (i.e., accelerometers), using the $v_{z}-v_{z}$ column of Table I, it is straightforward to obtain that (22) and (24) also apply.

\section{B. Combining Pressure and Particle Velocity}

The variance of the combination of pressure and particle velocity is, in general, given by

$$
\begin{aligned}
E\{ & \left.\left(P(\omega)+V_{z}(\omega)\right)\left(P^{*}(\omega)+V_{z}^{*}(\omega)\right)\right\} \\
= & E\left\{P(\omega) P^{*}(\omega)\right\}+E\left\{V_{z}(\omega) V_{z}^{*}(\omega)\right\} \\
& +2 \operatorname{Re}\left\{E\left\{P(\omega) V_{z}^{*}(\omega)\right\}\right\} .
\end{aligned}
$$

Herein, two cases should be considered: the pressure and the particle velocity are measured by collocated independent sensors or the pressure and the particle velocity are obtained from pressure measurements at closely located hydrophones. In the first case, for narrowband signal and noise, and using the same reasoning as above, it is straightforward to obtain the SNR at the output as

$$
\begin{aligned}
\mathrm{SNR}_{\mathrm{out}} & =\frac{S_{s}(\omega)\left(1+\sin ^{2}\left(\phi_{s}\right)\right)+2 S_{s}(\omega) \sin \left(\phi_{s}\right)}{S_{n}(\omega)\left(1+\sin ^{2}\left(\phi_{n}\right)\right)+2 S_{n}(\omega) \sin \left(\phi_{n}\right)} \\
& =\frac{\left(1+\sin \left(\phi_{s}\right)\right)^{2}}{\left(1+\sin \left(\phi_{n}\right)\right)^{2}} \mathrm{SNR}_{\mathrm{ps}} .
\end{aligned}
$$

It should be noted that, as for the particle velocity case discussed above, the SNR of a narrowband signal in directive noise depends on the directions of signal and noise. High SNR is 
achieved if the noise arrives from low elevation angles (close to the horizontal) and signal from steeper elevation angles, which occurs in seismic setups when the main source of noise is tow ship noise. Moreover, the directivity factor $(1+\sin (\phi))$ weights differently signals coming from positive and negative directions allowing to attenuate signals arriving from negative angles.

When the noise is 3-D isotropic, the $K_{p-v_{z}}$ is 0 (see Table I), and the output SNR is given by

$$
\mathrm{SNR}_{\mathrm{out}}=\frac{\left(1+\sin \left(\phi_{s}\right)\right)^{2}}{1+1 / 3} \mathrm{SNR}_{\mathrm{ps}} .
$$

Thus, for angles steeper than $\arcsin (\sqrt{1+1 / 3}-1)$, i.e., for $\phi_{s}>9^{\circ}$, the SNR at the VS output is higher than at the pressure sensor output.

When the particle velocity is obtained from closely spaced pressure sensors and approximations (5) and (9) hold, it can be seen that (26) also applies.

For the 3-D isotropic noise, the derivation of noise power at the sensors' output is also based on (25). The first term, related to the noise component of the acoustic pressure $P_{n}(\omega)$, is given by

$$
\begin{aligned}
E\left\{P_{n}(\omega) P_{n}^{*}(\omega)\right\} & =\frac{1}{2}\left\{S_{n}(\omega)+\operatorname{Re}\left\{S_{n}(\omega)\right\} \operatorname{sinc}(k D)\right\} \\
& \approx S_{n}(\omega)
\end{aligned}
$$

since for small $k D, \operatorname{sinc}(k D) \approx 1$. The term related to the cross power between pressure and particle velocity is given by

$$
\begin{aligned}
E & \left\{P_{n}(\omega) V_{z, n}^{*}(\omega)\right\} \\
= & \frac{1}{2 j k D} E\left\{\left(P_{1}(\omega)+P_{2}(\omega)\right)\left(P_{1}^{*}(\omega)-P_{2}^{*}(\omega)\right)\right\} \\
= & \frac{1}{2 j k D}\left\{E\left\{P_{1}(\omega) P_{1}^{*}(\omega)\right\}-E\left\{P_{2}(\omega) P_{2}^{*}(\omega)\right\}\right. \\
& \left.\quad-2 j \operatorname{Im}\left\{E\left\{P_{1}(\omega) P_{2}^{*}(\omega)\right\}\right\}\right\} \\
= & \frac{1}{2 j k D}\left\{S_{n}(\omega)-S_{n}(\omega)-2 j \operatorname{Im}\left\{S_{n}(\omega)\right\} \operatorname{sinc}(k D)\right\}=0
\end{aligned}
$$

because $\operatorname{Im}\left\{S_{n}(\omega)\right\}=0$.

Using the reasoning as for (23), the particle velocity noise power can be written as

$$
\mathrm{E}\left\{V_{z, n}(\omega) V_{z, n}^{*}(\omega)\right\} \approx \frac{S_{n}(\omega)}{3} .
$$

Therefore, summing (28)-(30), we conclude that (27) also applies when the combination of pressure and particle velocity is obtained from the measurements of two closely spaced hydrophones.

\section{Combining Particle Velocity and Particle Velocity Gradient}

Let us analyze the SNR at the output of the particle velocity gradient, obtained from a pair of closely located particle velocity sensors. The particle velocity gradient is given by (13); therefore, taking into account the analysis of the pressure difference, it can be shown that the SNR for the narrowband directional noise is

$$
\mathrm{SNR}_{\mathrm{out}}=\frac{\sin ^{4}\left(\phi_{s}\right)}{\sin ^{4}\left(\phi_{n}\right)} \mathrm{SNR}_{\mathrm{ps}}
$$

Similar to the pressure difference case, the particle velocity gradient improves the SNR when the noise arrives from directions close to the normal of the sensors' axis and the signal arrives from steeper elevation angles-what is usually the case in the seismic setup. The major difference between both combinations is the higher noise (signal) suppression of particle velocity gradient, when the noise (signal) direction deviates from the sensors' axis-which is clearly seen from the normalized amplitude directivity pattern presented in Fig. 2.

Applying the same reasoning as for the combination of pressure and pressure difference, the SNR for the case of directional noise with combination of particle velocity and particle velocity gradient using (15) is given by

$$
\mathrm{SNR}_{\text {out }}=\frac{\left(\sin \left(\phi_{s}\right)+\sin ^{2}\left(\phi_{s}\right)\right)^{2}}{\left(\sin \left(\phi_{n}\right)+\sin ^{2}\left(\phi_{n}\right)\right)^{2}} \mathrm{SNR}_{\mathrm{ps}}
$$

thus showing also an improved gain when the noise and the signal have opposite signs of elevation angles.

When the noise is 3-D isotropic, then the variance of the particle velocity gradient component of the noise is similar to (19) and is given by

$$
\begin{aligned}
& \mathrm{E}\left\{\bar{V}_{z, n}(\omega) \bar{V}_{z, n}^{*}(\omega)\right\} \\
&=\frac{1}{(k D)^{2}} \mathrm{E}\left\{V_{1}(\omega) V_{1}^{*}(\omega)+V_{2}(\omega) V_{2}^{*}(\omega)\right. \\
& \quad-2 \operatorname{Re}\left\{V_{1}(\omega)\left(V_{2}^{*}(\omega)\right\}\right\} .
\end{aligned}
$$

Using the values on Table I, one can write

$$
\begin{aligned}
\mathrm{E} & \left\{\bar{V}_{z, n}(\omega) \bar{V}_{z, n}^{*}(\omega)\right\} \\
& =\frac{1}{(k D)^{2}}\left\{\frac{2}{3} S_{n}(\omega)-2 S_{n}(\omega)\left\{\frac{J_{1}(k D)}{k D}-J_{2}(k D)\right\}\right\} .
\end{aligned}
$$

Using a Taylor expansion, it can be shown that for small $k D$, $J_{1}(k D) / k D-J_{2}(k D) \approx 1 / 3$, becoming the 3 -D isotropic noise power at the output of the particle velocity gradient approximately 0 . So, the overall noise is limited by the nonacoustic noise.

For the variance of the noise component of the combination of the particle velocity and particle velocity gradient, one can write

$$
\begin{aligned}
\mathrm{E}\left\{\tilde{V}_{z, n} \tilde{V}_{z, n}^{*}\right\} \\
=\mathrm{E}\left\{\left(V_{z, n}(\omega)+\bar{V}_{z, n}(\omega)\right)\left(V_{z, n}(\omega)+\bar{V}_{z, n}(\omega)\right)^{*}\right\} \\
=\mathrm{E}\left\{V_{z, n}(\omega) V_{z, n}^{*}(\omega)+\bar{V}_{z, n}(\omega) \bar{V}_{z, n}^{*}(\omega)\right. \\
\left.\quad+2 \operatorname{Re}\left\{V_{z, n} \bar{V}_{z, n}^{*}\right\}\right\} .
\end{aligned}
$$




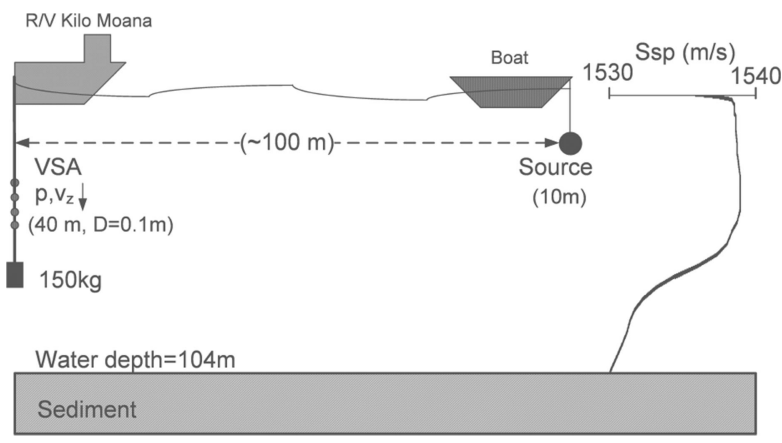

Fig. 5. MakaiEx'05 scenario: The source was deployed at 10-m depth within $100-\mathrm{m}$ range from the receiver. The VS was deployed at 40-m depth. The measured sound-speed profile (SSP) is shown on the right-hand side. The bottom parameters are given in Table II.

Since two particle velocity measurements $V_{1}(\omega)$ and $V_{2}(\omega)$ are used, one can write for the cross-power term

$$
\begin{aligned}
& \mathrm{E}\left\{\left(V_{z, n}(\omega) \bar{V}_{z, n}^{*}(\omega)\right\}\right. \\
&=\mathrm{E}\left\{\left(\frac{V_{1}(\omega)+V_{2}(\omega)}{2}\right)\left(\frac{V_{1}(\omega)-V_{2}(\omega)}{j k D}\right)^{*}\right\} \\
&= \frac{j}{2 k D} \mathrm{E}\left\{V_{1}(\omega) V_{1}^{*}(\omega)-V_{2}(\omega) V_{2}^{*}(\omega)\right. \\
&\left.\quad+2 \operatorname{Im}\left\{V_{1}(\omega) V_{2}^{*}(\omega)\right\}\right\} \\
&=\frac{j}{2 k D}\left\{S_{n}(\omega)-S_{n}(\omega)+0\right\}=0 .
\end{aligned}
$$

Thus, for the case of a narrowband signal and the 3-D isotropic noise, one can write

$$
\mathrm{SNR}_{\mathrm{out}}=\sin ^{4}\left(\phi_{s}\right) \frac{3}{2} \mathrm{SNR}_{\mathrm{ps}}
$$

From the discussion above, it is important to remark the limitations of VSs to detect signals in low SNR conditions, which imposes stringent requirements for sensor self-noise, particularly at low frequencies. Nevertheless, it can be concluded that the use of triorthogonal axis accelerometers located along the vertical axis will improve the processing gain of pressure and VS combinations in presence of directive noise from horizontal and surface directions, by steering the axis of maximum response to the bottom returns.

\section{Simulations}

The simulations are based on the experimental setup of the Makai 2005 Experiment (MakaiEx'05), which took place off the west coast of Kauai Island, HI, USA, in September 2005. For a trial description, see [32]. The pressure and the horizontal and the vertical particle velocity fields were computed with the oasp module of OASES [33] according to the MakaiEx'05 scenario depicted in Fig. 5, and bottom parameters of Table II. The source was within 100-m range from the receiver, but the simulations were generated for a source range of $100 \mathrm{~m}$. The signal is broadband $(0.5-1.5 \mathrm{kHz})$ with the spectrum shaped by a Hanning window. It should be noted that the theory was derived for one frequency $\omega$, but the same analysis could be
TABLE II

BotTom PARAMETERS USED IN SIMULATIONS FOR THE MAKAIEX'05 AREA, ESTIMATED IN [19]

\begin{tabular}{lccc}
\hline \hline Sediment & First layer & Second layer & Sub-bottom \\
\hline Thickness $(\mathrm{m})$ & 1.2 & 20 & - \\
$\rho\left(\mathrm{g} / \mathrm{cm}^{3}\right)$ & 1.65 & 1.9 & 2.1 \\
$c_{p}(\mathrm{~m} / \mathrm{s})$ & 1575 & 1671 & 2300 \\
$c_{S}(\mathrm{~m} / \mathrm{s})$ & 70 & 100 & 800 \\
$\alpha_{p}(\mathrm{~dB} / \lambda)$ & 0.5 & 0.6 & 0.1 \\
$\alpha_{S}(\mathrm{~dB} / \lambda)$ & 1.0 & 2.0 & 0.2 \\
\hline \hline
\end{tabular}

applied for a broadband signal using Fourier synthesis. The combination of pressure and particle velocity was computed using (8), where the vertical component of the particle velocity was given by the model or estimated from the pressure using (6). The combination of particle velocity and particle velocity gradient was computed using (15) from the vertical particle velocity given by the model. To compute the gradients, either for pressure or for particle velocity, the respective measurements were considered $10 \mathrm{~cm}$ apart. Then, the various time-domain arrival patterns were obtained as the envelope of the inverse Fourier transform.

Fig. 6(a) shows the arrival patterns (normalized amplitudes) for the pressure (blue) and the various combinations: pressure and particle velocity from pressure gradient (green); pressure and particle velocity given by the model (black); and particle velocity and particle velocity gradient (red). For amplitude comparison, the combinations of pressure and particle velocity and particle velocity and particle velocity gradient are divided by 2 . Both combinations of pressure and particle velocity overlap (green and black lines) and cannot be distinguished in the plot.

In Fig. 6(a), three groups of arrivals can be identified: the first group includes the direct and surface-reflected arrivals; the second group includes the bottom and surface-bottom-reflected arrivals; and the third group includes the bottom-surface and the surface-bottom-surface-reflected arrivals. In the first and third groups, the combination arrivals (red, black, and green) are significantly attenuated (more than 65\%) compared to the pressure-only arrival (blue), whereas in the second group, the amplitudes of the combination arrivals and the pressure-only arrival are almost identical. For the direct and surface-reflected arrivals in the first group, the combination of particle velocity and particle velocity gradient provides a much higher attenuation (around 90\%) than the combination of pressure and particle velocity, as can be expected from the directivity patterns in Fig. 2. For both combinations, the bottom reflections in the second group, arriving from angles close to the axis of maximum response, have similar amplitudes, whereas the surface arrivals in the third group are almost completely suppressed. It is worth mentioning that the green and black curves overlap as the combinations of pressure and particle velocity from pressure only and from pressure and particle velocity measurements are equivalent.

The spatial filtering capabilities of VS is enhanced when using particle velocity measurements from orthogonal direc- 


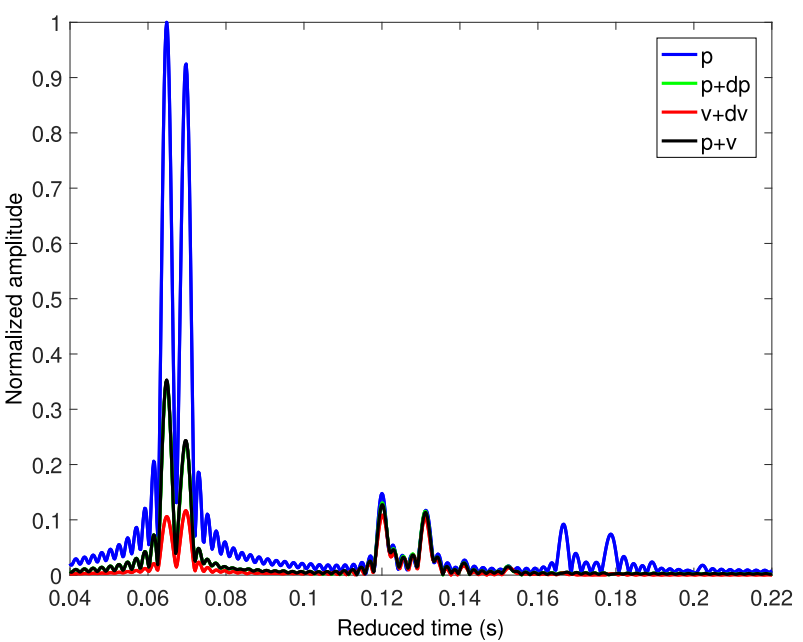

(a)

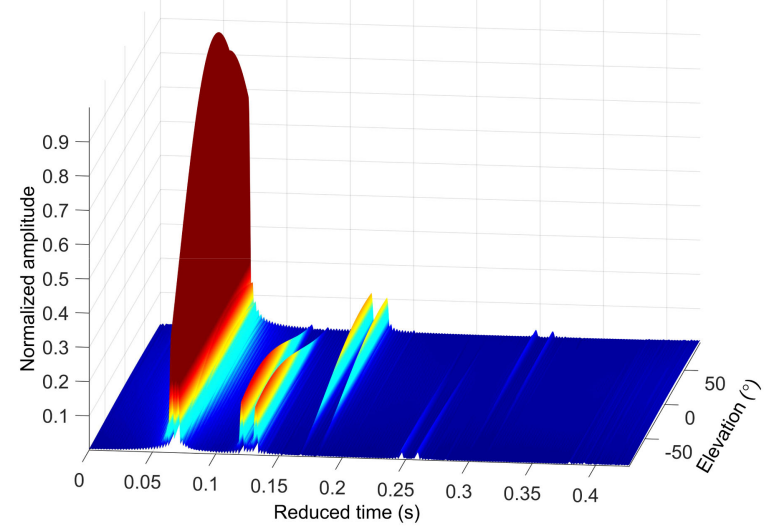

(b)

Fig. 6. Arrival patterns (normalized amplitudes) computed from signals generated by the oasp module of OASES, for the scenario depicted in Fig. 5 and bottom parameters of Table II considering (a) pressure only (blue), combination of pressure and pressure gradient (green), combination of particle velocity and particle velocity gradient (red), and combination of pressure and particle velocity (black); and (b) combination of pressure and vertical and horizontal components of particle velocity steered for elevation angles between $-90^{\circ}$ (bottom) and $90^{\circ}$ (surface).

tions. Fig. 6(b) shows the 2-D arrival patterns steered to various elevation angles computed from the combination of pressure and vertical and horizontal particle velocity using (17). Direct and surface reflections' arrivals impinge from positive elevation angles and bottom reflections impinge from negative elevation angles. This simple steering process allows the separation of the arrivals from the various directions keeping the relative amplitudes.

A simulation was performed to illustrate the behavior of the proposed methods in presence of diffuse noise coming from horizontal directions and tonal interferences from the sea surface close to the receiver, aimed at representing shipping noise and tow ship interference, respectively. Fig. 7 shows the obtained results in the same scenario as that of Fig. 6 for the broadband horizontal noise (same band and power as the signal) at 200-m range from the receiver and $10 \mathrm{~m}$ depth [Fig. 6(a)] and the sur-

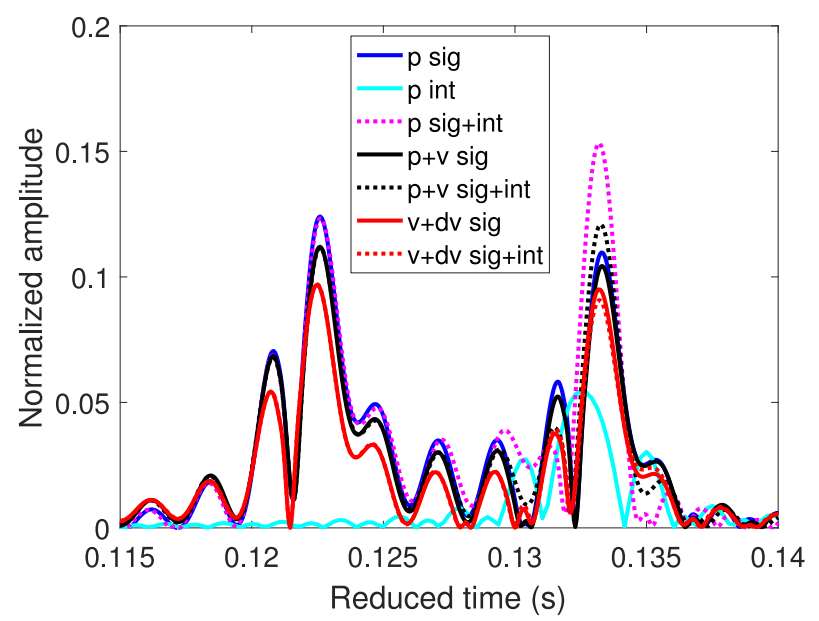

(a)

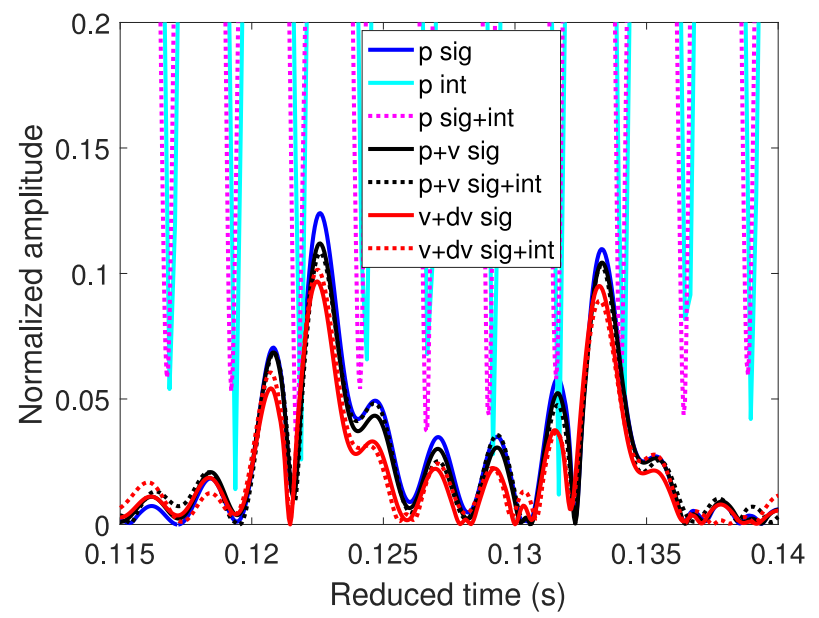

(b)

Fig. 7. Detail of the bottom-reflected arrivals (second group) computed by the oasp module of OASES as in Fig. 6, (a) for the scenario depicted in Fig. 5 and bottom parameters of Table II and the direct arrival of an broadband noise from horizontal direction with the same spectra and amplitude as the signal at 200 - $\mathrm{m}$ range; and (b) a narrowband tonal interference $(1000 \mathrm{~Hz})$ with an emitted power $40 \mathrm{~dB}$ higher than that of the signal and situated just above the receivers: pressure interference only (cyan); pressure of signal only (blue); pressure of signal and interference (dotted magenta); combination of pressure and particle velocity of signal (black); combination of signal and interference (dotted black); combination of particle velocity and particle velocity gradient of signal (red); combination of signal and interference (dotted red).

face tonal interference at $1000 \mathrm{~Hz}$ and $40 \mathrm{~dB}$ higher than the signal [Fig. 6(b)]. Only the group of bottom-reflected arrivals between reduced time 0.115 and $0.14 \mathrm{~s}$ is shown. The received pressure signal is represented by the blue lines, whereas broadband horizontal noise and tonal interference are represented by cyan lines. The magenta dotted lines represent the arrival patterns for the pressure obtained from the addition of the signal and broadband horizontal noise or tonal interference. The arrival patterns for the combination of pressure and particle velocity and the combination of particle velocity and particle velocity gradient are represented by black and red lines, respectively. Solid lines represent noise/interference free signal patterns as in the previous case for reference, and dotted lines represent arrival patterns with added broadband horizontal noise/tonal interference. 
In the broadband horizontal noise case [see Fig. 7(a)], the direct arrival of the interference overlaps with the bottom-reflected arrivals from the signal. The broadband horizontal noise changes significantly the amplitude of the bottom reflection (pressure) at approximately $0.133 \mathrm{~s}$, but has no influence in the peak at approximately $0.123 \mathrm{~s}$. The noise increases the highest peak amplitude by $3 \mathrm{~dB}$ for the pressure, $1.3 \mathrm{~dB}$ for the combination of pressure and particle velocity, and only $0.4 \mathrm{~dB}$ for the combination of particle velocity and particle velocity gradient. Moreover, the broadband horizontal noise changes significantly the lower amplitude peaks of pressure between the nulls at approximately 0.1275 and 0.1375 . These peaks may represent low amplitude returns from bottom layers, therefore containing relevant information for bottom characterization. It can be seen that the combination of pressure and particle velocity and, particularly, the combination of particle velocity and particle velocity gradient attenuates significantly the broadband horizontal noise on these peaks.

In the narrowband case [see Fig. 7(b)], the interference tone at $1000 \mathrm{~Hz}$ is $40 \mathrm{~dB}$ above the signal component at this frequency. It can be seen that the structure of the pressure arrival pattern is completely lost due to the strong tonal interference, but the interference is almost completely suppressed on the arrival patterns of combinations of pressure and particle velocity and particle velocity and particle velocity gradient.

In practice, the interferences might be a mix of the limiting cases considered above, but the advantage of VSs in suppressing such noise is clearly illustrated in these examples.

\section{EXPERIMENTAL DATA ANALYSIS}

The data analyzed here were acquired on September 25th, 2005, during the field calibration event of the MakaiEx'05 experiment in a setup similar to the scenario used in the simulations (see Fig. 5). The experimental receiving system consisted of four Wilcoxon TV-001 VSs, with available particle velocity (accelerometers) and pressure channels for data acquisition [3], [34], [35]. The sensors were configured in a vertical array with 10 -cm element spacing. The system was suspended off the stern of the research vessel Kilo Moana, with a 150-kg weight at the bottom, to ensure that the array stayed as close to the vertical as possible. The $z$-axis is vertically oriented downward, with the deepest sensor at $40 \mathrm{~m}$. The VSs are numbered from the shallowest 1 to the deepest 4 .

The sound source used in this experiment was a Lubell transducer model 916C, which was deployed at 10-m depth from a small auxiliary boat. The sound source transmitted sequences of linear frequency modulated chirps, multitones and m-sequences in the $0.5-14-\mathrm{kHz}$ band. The signals were acquired at a sampling frequency of $48 \mathrm{kHz}$. The Lubell source was towed during a period of $1 \mathrm{~h}$ from a distance of $2.5 \mathrm{~km}$ toward the research vessel that was holding a fixed position. The source localization from the LFM chirps in the $8-14-\mathrm{kHz}$ band and a single VS are reported in [36], where it is shown that the source at minute 56 from event start (data analyzed herein) is within the 100$\mathrm{m}$ range from the receiver. For the present analysis, 4.5-s-long LFM chirps spanning the $0.8-1.25-\mathrm{kHz}$ band were used.

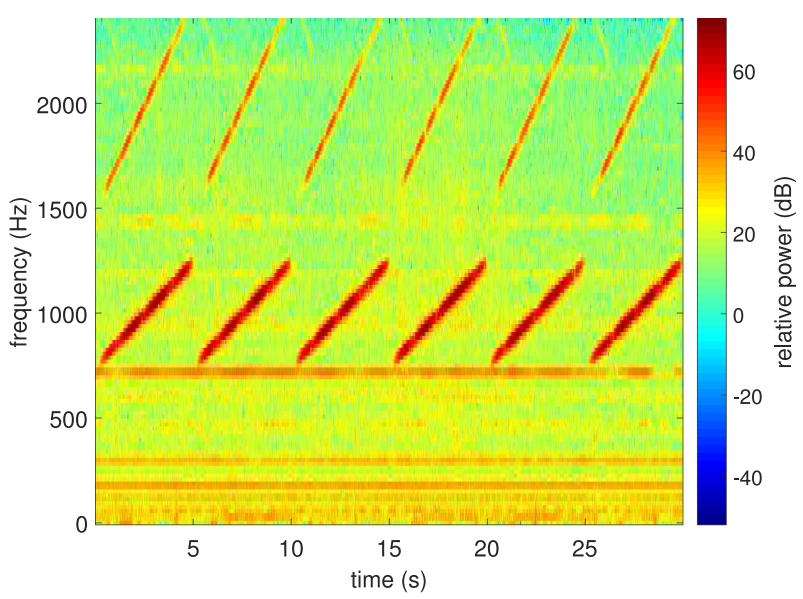

(a)

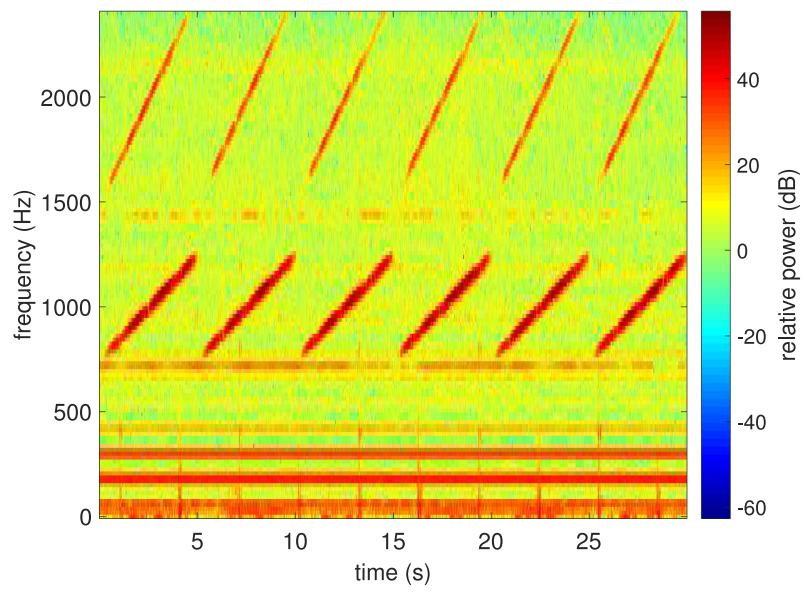

(b)

Fig. 8. Spectrograms of the signals received at minute 56 from event start during MakaiEx'05, at the shallowest VS: (a) pressure component; and (b) $z$-axis (vertical) particle velocity component.

Fig. 8 shows the spectrograms of the pressure [Fig. 8(a)] and the $z$-axis particle velocity [Fig. 8(b)] waveforms received at the shallowest VS. The signals were decimated to $8 \mathrm{kHz}$. For further processing, the acquired signals were filtered in the band $0.5-1.5 \mathrm{kHz}$ by a bandpass linear phase filter to remove the low-frequency ship noise and high-frequency harmonics of the transmitted signal (these harmonics are shown in Fig. 8 at frequencies greater than $1500 \mathrm{~Hz}$ ). Then, the various channels were cross correlated with the emitted source signal and combined in the frequency domain to obtain the various arrival patterns.

The results are shown in Fig. 9, where the envelopes of the arrivals were obtained from pressure measurements only [Fig. 9(a)] and particle velocity measurements only [Fig. 9(b)]. Fig. 9(c) compares the combination of pressure and particle velocity from pressure measurements only with the combination of pressure and particle velocity measurements. It should be remarked that for pressure comparison purposes, the amplitudes of the curves of the combination of pressure and particle velocity and the combination of particle velocity and particle velocity gradient were divided by 2 .

Three groups of arrivals can be identified in the various arrival patterns: the first group includes the direct and surface-reflected 


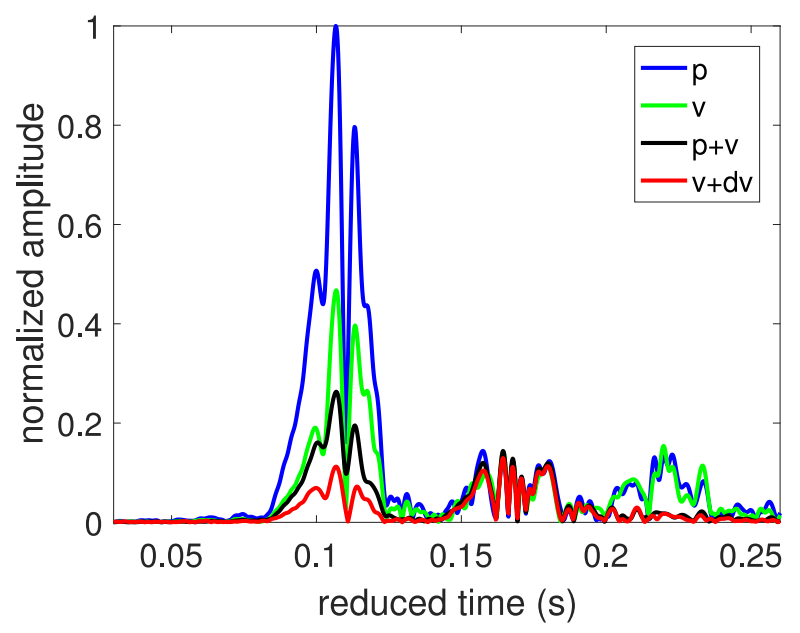

(a)

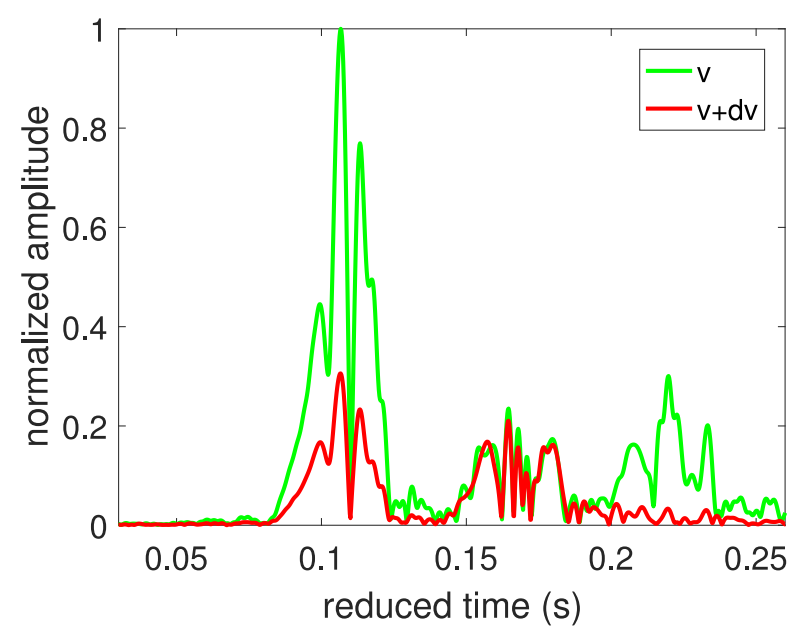

(b)

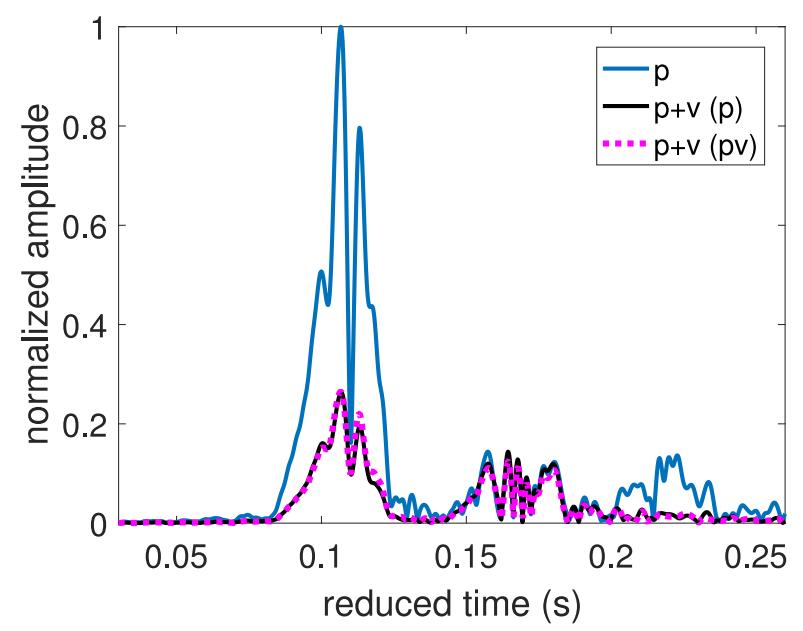

(c)

Fig. 9. Arrival patterns (normalized amplitudes) computed from signals received at minute 56 from run start of MakaiEx'05 using (a) pressure measurements only (blue - pressure; green — particle velocity; black—combination of pressure and particle velocity; red - combination of particle velocity and particle velocity gradient); (b) particle velocity measurements only (green-particle velocity, red - combination of particle velocity and particle velocity gradient); and (c) both pressure and particle velocity channels [dotted magenta-combination of pressure and particle velocity from particle velocity channel, blue and black same as (a)]. arrivals; the second group includes the bottom-reflected arrivals; and the third group includes the bottom-surface-reflected arrivals. Comparing the simulations of Fig. 6 with the data of Fig. 9, additional equal amplitude arrivals can be noticed, possibly due to bottom and sub-bottom layers, which suggests that the real environment has a stronger reflector than in the model. In the bottom-surface-reflected arrivals (third group), this structure is smeared, most likely because the roughness of sea surface reduces the coherence of the bottom-reflected arrivals.

As aforementioned, in Fig. 9(a), all curves were obtained from pressure channels only. The blue curve was computed from the average of the pressure channels at VSs 1 and 2. The green curve is the particle velocity computed by the pressure gradient from VSs 1 and 2 [using (4)], whereas the black curve is the combination of pressure and particle velocity [using (10)]. The red curve was computed from the pressure channels using (15) where $V_{1}(\omega)$ and $V_{2}(\omega)$ were obtained from the pressure gradient of pressure channels of VSs 1 and 2 and VSs 2 and 3, respectively. It can be noticed that the combination of pressure or vertical particle velocity with respective gradients significantly attenuates the surface arrivals, which is best seen with the latter combination (red) with more than $70 \%$ attenuation. From the peaks related to the direct arrival of the particle velocity and the pressure and (7), the elevation of the direct arrival is estimated by $\arcsin \left(A_{v} / A_{p}\right)$, where $A_{v}$ and $A_{p}$ are the peaks related particle velocity and pressure, respectively. The value obtained is approximately $27.9^{\circ}$. Using the ratio between the combination of pressure and particle velocity and pressure $\left(A_{p v} / A_{p}, A_{p v}\right.$ is the peak related to the combination of pressure and particle velocity) and (8), the estimate of the elevation angle $\phi$ is $28.7^{\circ}$, given by the solution of $|1+\sin (\phi)| / 2=A_{p v} / A_{p}$, where the factor 2 is due to the normalization considered in Fig. 9. Similarly, from the ratio between the combination of particle velocity and particle velocity gradient and the combination of particle velocity and pressure $\left(A_{v d v} / A_{p v}, A_{v d v}\right.$ is the peak related to the combination of particle velocity and particle velocity gradient) and combining (8) and (15), the elevation angle of the direct arrival is estimated by $\arcsin \left(A_{v d v} / A_{p v}\right)$, giving a value of $25.2^{\circ}$. Since the estimates from particle velocity and the combination of pressure and particle velocity are computed using the same pair of pressure channels, it is not surprising that the estimated angles of arrival are very close. The combination of the particle velocity and particle velocity gradient uses a third pressure channel, which may explain the approximately $3^{\circ} \mathrm{de}-$ viation from the particle velocity and combination of pressure and particle velocity estimates, since the amplitude among pressure channels varied as high as $3 \mathrm{~dB}$. The source-receiver range estimated by simple geometry using a source depth of $10 \mathrm{~m}$ and a receiver depth of $40 \mathrm{~m}$ is $56 \mathrm{~m}$ for an angle of arrival of $28^{\circ}$ and $64 \mathrm{~m}$ for an angle of arrival of $25^{\circ}$. These estimates are consistent with the expected source track [36].

The arrival patterns obtained from the vertical component ( $z$ axis) of particle velocity channels only are shown in Fig. 9(b). The green curve was computed from VS 1, and the red curve was computed from VSs 1 and 2 (using (15), divided by 2 for consistency with pressure results). The curves are normalized by the maximum of the particle velocity. It can be noticed that 
the direct and surface-reflected arrivals are more attenuated by the combination of particle velocity and particle gradient than by the particle velocity only. The elevation angle of the direct arrival estimated from the ratio between related peaks of the curves by combining (8) and (15), given by the solution of $|1+\sin (\phi)| / 2=A_{v d v} / A_{v}$, is $23^{\circ}$ and the corresponding source-receiver range is $71 \mathrm{~m}$. This result is in close agreement with the source-receiver range of $70 \mathrm{~m}$ estimated from the travel time difference between the direct and surface-reflected arrivals assuming a constant sound speed of $1540 \mathrm{~m} / \mathrm{s}$. The bottom reflections (second group of arrivals) estimated by the various sensor combination methods give very similar results; nevertheless, the combination of particle velocity and particle velocity gradient is slightly smaller, as this combination shows higher suppression for signals departing from $90^{\circ}$. The higher suppression of the combination of particle velocity and particle velocity outside of the bottom reflection group is also noticed, not only for the group of bottom-surface-reflected arrivals (third group) but also between direct and bottom-reflected group. The latter may be ascribed to a side-lobe reduction due to the surface-reflected arrival and/or the attenuation of noise from the surface.

In Fig. 9(c), the magenta curve (dotted) was computed by the combination of pressure and particle velocity from pressure and the $z$-axis particle velocity channels of the shallowest VS. This curve compares with black curve, which is the same as in Fig. 9(b), i.e., the combination of pressure and particle velocity from pressure only channels. Both curves are normalized by the maximum of the arrival pattern from the pressure (blue curve). However, a weight of $1 / 3$ was applied to particle velocity due to different gains in pressure and particle velocity channels. This tuning parameter does not strongly influence the results as seen by Fig. 3 in Section II-D. It can be noticed that the results of the combination of pressure and particle velocity, which is given by pressure gradient or by the $z$-axis particle velocity component, are coincident concluding that the particle velocity could be obtained from two pressure sensors or simply using a single particle velocity sensor (accelerometer). These results suggest that using a combination of one or two VS allows us to filter out the undesirable direct and surface-reflected echoes from the received waveforms by keeping the bottom-reflected echoes.

Taking advantage of the triorthogonal axis VS, a search for all possible azimuths and elevations was performed using (18) in time domain. The result presented in Fig. 10(a) as an ambiguity surface for the highest peak (direct path), located approximately at $\left(-150^{\circ}, 30^{\circ}\right)$, indicates the DOA of the direct path (the elevation is close to that given by the combination of pressure and particle velocity from pressure only channels described above). Therefore, this ambiguity surface corresponds to the time of arrival of the direct arrival. The 2-D slice (elevation time) presented in Fig. 10(b) corresponds to the azimuth at which the peak of the ambiguity surface in Fig. 10(a) is located. Comparing with Fig. 9, Fig. 10 highlights with real data the advantage of triorthogonal axis accelerometers, which clearly allows for the separation of bottom reflections (negative elevation angles) from direct and surface-reflections (positive elevation angles). The overall results also highlight the importance of the calibration of the various VS channels.

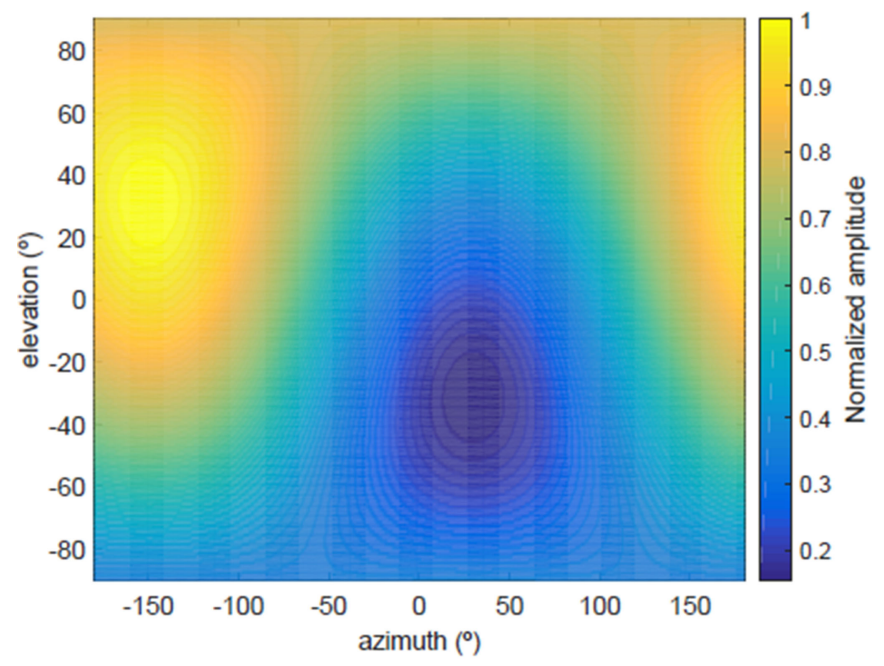

(a)

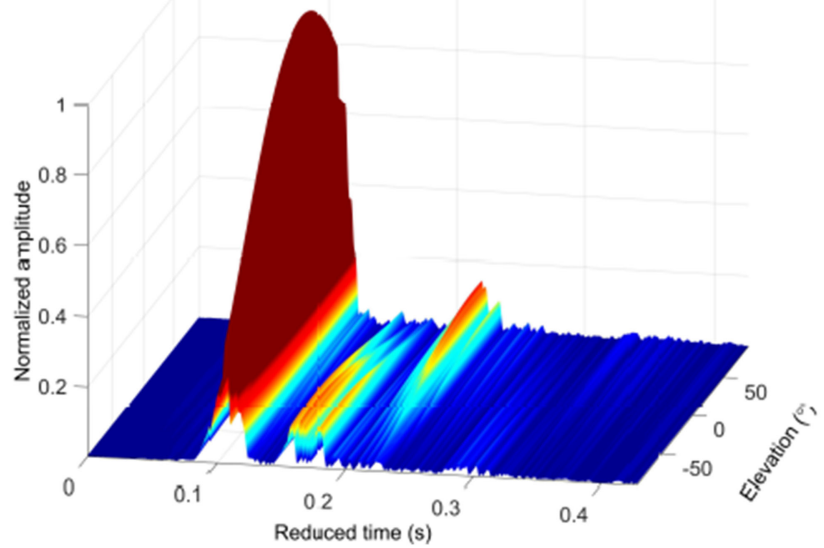

(b)

Fig. 10. (a) Azimuth-elevation normalized ambiguity surface obtained for the direct path (highest peak) using (18) in time domain; and (b) corresponding 2-D slice (elevation time) for the azimuth fixed at the peak value, at minute 56 from run start of the MakaiEx'05 sea trial. Elevation angle $+90^{\circ}$ points to the sea surface and $-90^{\circ}$ points to the bottom

\section{CONCLUSION}

The present study suggests that the combination of pressure and/or particle velocity in a single VS element allows for the separation of bottom returns from direct and surface reflections, which are undesired for seismic applications. The sensing element should, in this case, measure the pressure and the particle velocity by collocated independent sensors or by, at least, two pressure or particle velocity sensors (accelerometers), located along the vertical axis with a distance smaller than the one-fourth of the minimum wavelength. The SNR analysis of the various VS combinations shows that the combination of pressure with particle velocity or combination of particle velocity with particle velocity gradient improves the SNR of bottom reflections in presence of directional and correlated noise. The steering capabilities of the triorthogonal axis accelerometers combined with pressure were also discussed, not only for noise suppression 
but also to improve bottom reflections, important for seismic exploration.

The results were obtained with simulated data and validated with experimental data acquired during the Makai'05 Experiment. The 3-D DOA of the acquired arrival pattern structure was achieved, where the difference between the elevation angles due to the surface or bottom reflections could be clearly seen. The theoretical analysis and experimental data results prove that the use of a pressure-particle velocity combination allows us to cancel or significantly attenuate direct or surface reflections (approximately 6-10 dB), improving bottom reflections. In seismic exploration, the problem of filtering out undesirable direct and surface-reflect arrivals (deghosting) is improved by simply using the pressure combined with particle velocity measurements, given by pressure gradient or by accelerometers. These results suggest that size and autonomy of such sensor arrangements may advantageously replace or complement short AUV towed streamers for seismic imaging surveys, such as those planned in the WiMUST project funded under the H2020 research program of the European Union.

\section{ACKNOWLEDGMENT}

The authors would like to thank chief scientists M. Porter and P. Hursky from the HLS team, for participating in the MakaiEx'05 sea trial, funded by ONR, and also J. Tarasek from the Naval Surface Weapons Center for the loan of the vector sensor array and B. Abraham from Applied Physical Sciences for providing assistance with the data acquisition.

\section{REFERENCES}

[1] P. Felisberto, P. Santos, D. Maslov, and S. M. Jesus, "Combining pressure and particle velocity sensors for seismic processing," in Proc. MTS/IEEE/OES Oceans, Monterey, CA, USA, Sep. 2016, pp. 1-6.

[2] Reflection seismology. 2017. [Online]. Available: https://en.wikipedia. org/wiki/Reflection_seismology

[3] J. C. Shipps and B. M. Abraham, "The use of vector sensors for underwater port and waterway security," in Proc. Sensors Ind. Conf., New Orleans, LA, USA, Jan. 2004, pp. 41-44.

[4] C. Sherman and J. Butler, Transducers and Arrays for Underwater Sound (The Underwater Acoustic Series). New York, NY, USA: Springer, 2007.

[5] A. Nehorai and E. Paldi, "Acoustic vector-sensor array processing," IEEE Trans. Signal Process., vol. 42, no. 9, pp. 2481-2491, Sep. 1994.

[6] M. Hawkes and A. Nehorai, "Acoustic vector-sensor beamforming and capon direction estimation," IEEE Trans. Signal Process., vol. 46, no. 9, pp. 2291-2304, Sep. 1998.

[7] C. Wan, A. Kong, and C. Liu, "A comparative study of DOA estimation using vector/gradient sensors," in Proc. Oceans, May 16-19, 2007, pp. $1-4$.

[8] B. Cray and A. Nuttall, "Directivity factors for linear arrays of velocity sensors," J. Acoust. Soc. Amer, vol. 110, no. 1, pp. 324-331, Jul. 2001.

[9] G. D’Spain, W. Hodgkiss, G. L. Edmonds, J. C. Nickles, F. H. Fisher, and R. A. Harriss, "Initial analysis of the data from the vertical DIFAR array," in Proc. MTS/IEEE Oceans, Newport, RI, USA, Oct. 1992, pp. 346351.

[10] V. N. Hari, G. V. Anand, and A. B. Premkumar, "Narrowband signal detection techniques in shallow ocean by acoustic vector sensor array," Digit. Signal Process., vol. 23, pp. 1645-1661, Sep. 2013.

[11] M. Hawkes and A. Nehorai, "Acoustic vector-sensor correlations in ambient noise," IEEE J. Ocean. Eng., vol. 26, no. 3, pp. 337-347, Jul. 2001.
[12] G. D’Spain, J. Luby, G. Wilson, and R. Gramann, "Vector sensors and vector sensor line arrays: Comments on optimal array gain and detection," J. Acoust. Soc. Amer, vol. 120, no. 1, pp. 171-185, 2006.

[13] J. He and Z. Liu, "Two-dimensional direction finding of acoustic sources by a vector sensor array using the propagator method," Signal Process., vol. 88, pp. 2492-2499, 2008

[14] K. M. Krishna and G. V. Anand, "Narrowband detection of acoustic source in shallow ocean using vector sensor array," in Proc. MTS/IEEE Oceans, Biloxi, MS, USA, 2009, pp. 1-8.

[15] V. N. Hari, G. V. Anand, A. B. Premkumar, and A. S. Madhukumar, "Underwater signal detection in partially known ocean using short acoustic vector sensor array," in Proc. MTS/IEEE Oceans, Santander, Spain, Jun. 2011, pp. 1-9.

[16] P. Felisberto, P. Santos, and S. M. Jesus, "Traking source azimuth using a sin GLE vector sensor," in Proc. 4th Int. Conf. Sensor Technol. Appl., Venice, Italy, Jul. 2010, pp. 416-421.

[17] A. Abdi, H. Guo, and P. Sutthiwan, "A new vector sensor receiver for underwater acoustic communication," in Proc. MTS/IEEE Oceans, Vancouver, BC, Canada, 2007, pp. 1-10.

[18] A. Song, M. Badiey, P. Hursky, and A. Abdi, "Time reversal receivers for underwater acoustic communication using vector sensors," Quebec City, QC, Canada, Sep. 2008, pp. 1-10.

[19] P. Santos, O. C. Rodríguez, P. Felisberto, and S. M. Jesus, "Seabed geoacoustic characterization with a vector sensor array," J. Acoust. Soc. Amer. vol. 128, no. 5, pp. 2652-2663, Nov. 2010.

[20] H. Peng and F. Li, "Geoacoustic inversion based on a vector hydrophone array," Chin. Phys. Lett., vol. 24, no. 7, pp. 1997-1980, 2007.

[21] B. Nichols and K. G. Sabra, "Cross-coherent vector sensor processing for spatially distributed glider networks," J. Acoust. Soc. Amer., vol. 138, pp. EL329-EL335, Sep. 2015.

[22] D. Lindwall, "Marine seismic surveys with vector acoustic sensors," in Proc. SEG/New Orleans Ann. Meet., 2006, pp. 1208-1212.

[23] M. Widmaier, E. Fromyr, and V. Dirks, "Dual-sensor towed streamer: From concept to fleet-wide technology platform," First Break, vol. 33, pp. 83-89, 2015

[24] M. Widmaier and O. Lie, "Experiences with dual-sensor towed streamer acquisition and imaging in the eastern mediterranean," Search Discovery, Online J. Amer. Assoc. Petroleum Geologists, pp 1-6, Jul. 2016.

[25] H. Al-Khatib et al., "The widely scalable mobile underwater sonar technology (WIMUST) project: An overview," in Proc. OCEANS, May 2015, pp. 1-5.

[26] K. B. Smith and A. V. van Leijen, "Steering vector sensor array elements with linear cardioids and nonlinear hippioids," J. Acoust. Soc. Amer. vol. 122, no. 1, pp. 370-377, 2007.

[27] H. Cox, "Spatial correlation in arbitrary noise fields with application to ambient sea noise," J. Acoust. Soc. Amer, vol. 54, no. 5, pp. 1289-1301, 1973.

[28] W. A. Kuperman and F. Ingenito, "Spatial correlation of surface generated noise in a stratified ocean," J. Acoust. Soc. Amer., vol. 67, no. 6, pp. 1988 1996, 1980.

[29] E. Habets and S. Gannot, "Generating sensor signals in isotropic noise fields," J. Acoust. Soc. Amer, vol. 122, no. 6, pp. 3464-3470, 2007.

[30] A. Abdi and H. Guo, "Signal correlation modeling in acoustic vector sensor arrays," IEEE Trans. Signal Process., vol. 57, no. 3, pp. 892-903, Mar. 2009.

[31] A. Poulsen, "Robust vector sensor array processing and performance analysis," Ph.D. dissertation, Dept. Elect. Eng. Comput. Sci., Massachusetts Inst. Technol., Cambridge, MA, USA, 2009.

[32] M. Porter et al., "The Makai experiment: High frequency acoustics," in Proc. 8th Eur. Conf. Underwater Acoustic, Carvoeiro, Portugal, 2006, vol. 1215 , pp. $918-935$.

[33] H. Schmidt, "Oases-User guide and reference manual," Dept. Ocean Eng., Massachusetts Inst. Technol., Cambridge, MA, USA, Tech. Rep. Version 3.1, Oct. 2004.

[34] B. M. Abraham, "Ambient noise measurements with vector acoustic hydrophones," in Proc. MTS/IEEE Oceans, Boston, MA, USA, Sep. 2006.

[35] M. T. Silvia and R. T. Richards, "A theoretical and experimental investigation of low-frequency acoustic vector sensors," in Proc. MTS/IEEE Oceans, Biloxi, MS, USA, Oct. 2002, pp. 1886-1897.

[36] P. Felisberto, O. Rodriguez, P. Santos, E. Ey, and S. M. Jesus, "Experimental results of underwater cooperative source localization using a single acoustic vector sensor," Sensors, vol. 13, no. 7, pp. 8856-8878, 2013. 


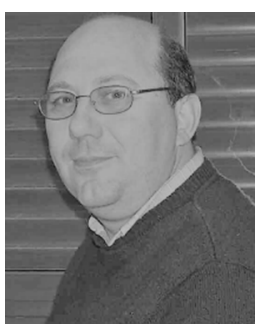

Paulo Felisberto was born in Portimão, Portugal. He received the Engineer degree in electrical engineering from Slovak Technical University, Bratislava, Slovakia, in 1988 and the M.Sc. and Ph.D. degrees in electronic and computer engineering from the University of Algarve, Faro, Portugal, in 1995 and 2005, respectively.

He is currently a Professor at the Department of Electrical Engineering, University of Algarve. He is a permanent member of the Signal Processing Laboratory (SiPLAB/CINTAL/UALG) at the University of Algarve and the Laboratory of Robotics and Systems in Engineering and Science (LARSyS/lisbon). He has participated and coordinated several projects financed by the Science and Technology Foundation-Portuguese Government and European Union. His main scientific interests include the field of signal processing in underwater acoustics with application in source localization and positioning and acoustic environmental monitoring.

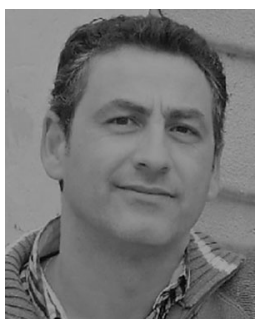

Paulo Santos was born in Oporto, Portugal. He received the B.S. degree in electromechanical engineering from the University of Beira Interior, Covilhã, Portugal, in 1994, the M.Sc. degree in electrical and computer engineering from the "Instituto Superior Técnico," Lisbon, Portugal, in 2000, and the Ph.D. degree in electronics and telecommunications engineering from the University of Algarve, Faro, Portugal, in 2012, with the topic "Ocean parameter estimation with high-frequency signals using a vector sensor array."

Since 1995, he has been with the "Instituto Superior de Engenharia (ISE)," University of Algarve, and is currently an Adjoint Professor with the Department of Electrical Engineering, ISE, where, among others, he teaches measurements and instrumentation and industrial instrumentation. He is a permanent member of the Signal Processing Laboratory (SiPLAB/CINTAL/UALG), University of Algarve, and a Research Member of the Laboratory of Robotics and Systems in Engineering and Science (LARSyS/Lisbon). He has participated in several Portuguese and European projects. His main research interests include underwater acoustics propagation, direction of arrival estimation, and vector sensors. He is an expert of 3-D array beamforming and source localization, and is working on the recent field of vector sensor array processing for geoacoustic inversion.

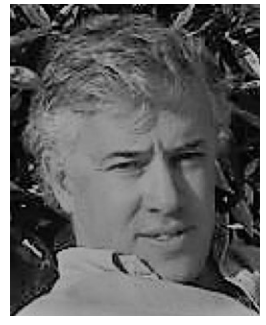

Sérgio M. Jesus (M'09) received the DoctoratesSciences degree in engineering sciences from the University of Nice, Nice, France, in 1986.

From 1985 to 1992, he was a Staff Scientist with the SACLANT Undersea Research Centre [NURC, currently Center for Maritime Research and Experimentation (CMRE)], La Spezia, Italy. During that period, he was involved with underwater acoustic field noise directionality and early studies on target detection using matched field processing. In 1992, he joined the Electronics and Computation Department, University of Algarve, Faro, Portugal, where he is currently a Full Professor. His research interests include underwater acoustic array signal processing, modelbased inversion, underwater communications, as well as other aspects dealing with sound propagation in the ocean, including its usage for environmental and biodiversity monitoring and conservation.

Prof. Jesus is a member of the IEEE Signal Processing Society, the European Association for Signal Processing, and the Acoustical Society of America. 